\title{
BEHAVIORAL AND ENVIRONMENTAL CORRELATES OF INFANT ABUSE IN GROUP. LIVING PIGTAIL MACAQUES
}

\author{
Dario Maestripieri \\ Emory University \\ Kelly A. Carroll \\ Berry College
}

\begin{abstract}
The present study investigated the context of occurrence of infant abuse and the behavior of abusive mothers and their infants in pigtail macaques (Macaca nemestrina). Subjects were 8 abusive mothers with their infants and 8 control mother-infant pairs living in 3 captive social groups. The most common forms of abuse were infant crushing and dragging and the most common context of occurrence was social stress. Severe and mild abuse differed in the frequency but not in the type of abuse patterns. Abusive mothers had controlling parenting styles relative to nonabusive mothers, and abused infants played at a later age and less frequently than controls. This study replicates previous findings on the parenting styles of abusive macaque mothers and provides new evidence on the context of occurrence of abuse and its consequences for infant health and social development.
\end{abstract}

\section{INTRODUCTION}

In Old World monkeys such as macaques (genus Macaca), mangabeys (Cercocebus), baboons (Papio) and vervet monkeys (Cercopithecus), young infants are totally dependent on their mothers for nutrition, transport, and protection from other conspecifics or preda- tors. Mothers are generally protective of their infants and restrict their activity in the first two months of life, and then gradually begin to reject infant contact and nursing attempts until infants are weaned, usually by the end of the first year (Altmann, 1980; Hinde \& Rowell, 1967). Rejection may cause infant distress and tantrums and the most persistent infants are

- Dario Maestripieri, Yerkes Regional Primate Research Center, Emory University, 2409 Taylor Lane, Lawrenceville, GA 30043; e-mail: psydm@emory.edu. 
occasionally rebuffed with slapping and biting by mothers.

Although most monkey mothers display only nurturant behavior towards their infants in their first months of life, a minority of mothers show violent behaviors such as infant dragging and throwing, pinning the infant down with their hands, and stepping or sitting on the infant. These patterns of behavior are morphologically distinct from maternal aggression in weaning conflicts and are best viewed as infant abuse (Troisi \& D'Amato, 1983). Infant abuse may occur as early as the first day of infant life and its consequences range from infant distress to serious injury and death (see Maestripieri \& Carroll, 1998a, for review).

Infant abuse has been observed in both mothers reared and housed in conditions of social deprivation (Reite \& Caine, 1983; Ruppenthal, Arling, Harlow, Sackett, \& Suomi, 1976) and mothers born and reared in social groups (hereafter group-living), in captivity and in the wild (Hiraiwa, 1981; Maestripieri, 1994a, 1998; Troisi, D'Amato, Fuccillo, \& Scucchi, 1982). Whereas infant abuse displayed by socially deprived monkeys is only one example of a host of abnormal social behaviors, group-living abusive mothers are otherwise normal individuals who possess and display a full range of competent social behaviors. Moreover, group-living abusive mothers also possess adequate caregiving skills, abuse being limited to only a small proportion of the interactions with their infants (e.g., Troisi \& D'Amato, 1984).

Studies of infant abuse in group-living macaques and mangabeys have revealed some similarities with child abuse in the prevalence of this phenomenon in large populations (510\%; Maestripieri, Wallen, \& Carroll, 1997a, 1997b), the intergenerational transmission of abuse (Maestripieri et al., 1997a), some psychological and behavioral characteristics of abusive parents (Maestripieri, 1998; Troisi \& D'Amato, 1984), and the role of psychosocial stress in triggering abuse (Maestripieri, 1994a). Therefore, the study of this phenomenon could provide information potentially use- ful to the understanding and prevention of child abuse (Maestripieri \& Carroll, 1998a). Infant abuse in group-living monkeys is a relatively infrequent phenomenon, however, and only one study has quantitatively investigated the behavior of abusive mothers and their infants with a sample size greater than one (Maestripieri, 1998).

In the present study we investigated several characteristics of infant abuse in group-living pigtail macaques including the rates of abuse and its severity, its most common forms and contexts of occurrence, and the developmental and previous parenting history of abusive mothers. We also compared the behavior of abusive mothers and their infants to that of control individuals to assess whether abusive behavior is associated with specific parenting styles and has negative consequences for early social development.

\section{METHOD}

\section{Subjects and Housing}

The subjects of this study were 16 pigtail macaque mothers with their infants living in three large social groups at the Field Station of the Yerkes Regional Primate Research Center in Lawrenceville, Georgia, U.S.A. Each group consisted of two to five adult males and 25-30 adult females with their immature offspring. The monkeys were housed in outdoor compounds $(25 \times 25 \mathrm{~m})$ with attached indoor quarters. They were fed twice a day and water was available as libitum.

Eight mothers were classified as abusive and eight as controls. The criterion used to identify abusive mothers was the occurrence of at least one of the following behavior patterns in the first days days of lactation: infant dragging, crushing, throwing, or sitting/stepping on (see below for definitions). These are the most commonly observed patterns of infant abuse in macaques (Maestripieri, 1998; Maestripieri et al., 1997a; Troisi \& D'Amato, 1983). Other abuse patterns such as hitting and 
biting were not used for the initial identification of abusive mothers because they sometimes occur also during mother-infant weaning conflicts. One mother was identified as abusive retrospectively, after she had been observed for the first 12 weeks of lactation. After the identification of abusive mothers, controls were individually matched to each of them for parity (primiparous or multiparous), dominance rank (high, middle, or low), and infant sex (male or female). In five cases, the subjects and their matched controls were from the same group and in three cases from different groups.

\section{Developmental and Parenting History of the Subjects}

Six abusive mothers and all controls were caught in the wild at the age of 2-4 years and arrived at the Yerkes Center about one year later. Two abusive mothers were born at the Yerkes Center and grew up in their natal group. The two captive-born subjects were mother and daughter and belonged to a family with an intergenerational history of infant abuse (Maestripieri et al., 1997a). The daughter suffered mild abuse during infancy while information on the early history of the mother was not available. Prior to this study, all animals had been used mostly for breeding purposes and observational studies of behavior and had not been used for invasive experimental studies or removed from their social group for long periods other than for medical reasons. Three abusive mothers were primiparous and one had one previous surviving infant. Four abusive mothers had a history of severe infant abuse, having caused death or permanent removal of most of their previous infants $(5 / 5,4 / 5,3 / 4$, and $6 / 11)$. Three control mothers were primiparous and none of the other multiparous mothers had abused their previous infants.

\section{Data Collection and Analysis}

Observations of subjects and controls began in the first week of infant life and ended in the 12th week, unless the infant died or had to be permanently removed due to abuse. Mother-infant pairs were focally observed in four weekly 30-min observation sessions randomly distributed between 0800 and $1900 \mathrm{hr}$. The mothers who severely abused their previous infants, however, were observed for up to 5 hours per week in the first two weeks of lactation. Observations were made from a platform that provided unrestricted view of the entire compound and behavioral data were collected with a portable computer. Data collection included infant abuse and other interactions between mothers and infants, between mothers and other individuals, and between infants and other individuals.

\section{Infant Abuse}

The following maternal behavior patterns were classified as infant abuse: (1) dragging: the mother drags her infant by its tail or leg while walking or running; (2) crushing: the mother pushes her infant on the ground with both hands; (3) throwing: the mother throws her infant at a short distance with one hand while standing or walking; (4) hitting: the mother violently slaps her infant with one hand or arm; (5) biting; (6) stepping or sitting on: the mother steps on her infant with one foot or both feet, or sits on her infant; (7) dangling/dropping: the mother climbs a tree or fence and holds her infant by its tail or leg or drops her infant on the ground. Infant abuse was scored independently of all the other mother-infant interactions (e.g., rejection). Abuse episodes did not last more than a few seconds and therefore only their frequency was recorded. Infant abuse was recorded as two separate events if there was a transition in the pattern of behavior (e.g. from dragging to throwing) or if there was a pause of at least 10 s during the behavior.

\section{Mother-Infant Interactions}

The following measures were used: (1) percentage of time spent in contact; (2) frequency 
of contacts made and broken by mothers and infants; (3) frequency of approaches and leaves by mothers and infants. Approaches and leaves were scored in relation to proximity within arm's reach (about $60 \mathrm{~cm}$ ); (4) percentage of all infant attempts to break contact that were prevented by the mother by pulling the infant by its tail or leg (restraining); (5) percentage of all infant attempts to make contact that were prevented by the mother by turning, running away, or holding the infant at a distance with an arm (rejection); rejection also included forcible removal from the mother's nipple; (6) percentage of contact time in which the mother kept her arms around the infant (cradling); (7) percentage of contact time in which the mother groomed her infant.

\section{Mother-Others Interactions}

The following measures were used: (1) frequency of contacts made and received by mothers; (2) frequency of approaches made and received by mothers (in relation to proximity within arm's reach); (3) frequency and duration of grooming done and received by mothers; (4) frequency of aggressive acts (threats, bites, and chases) done and received by mothers.

\section{Infant-Others Interactions}

The following measures were used: (1) frequency of infant handling (touching, carrying) and harassment (pulling, dragging, hitting, biting associated with infant screams) episodes initiated by other individuals; (2) frequency of social play (rough-and-tumble or chase) between infants and other infants or juveniles. Infant kidnapping occurred when another adult female snatched an infant and did not return it to its mother for more than $5 \mathrm{~min}$.

Behavioral comparisons between subjects and their matched controls were made using Student's $t$ tests for paired samples. For surviving infants and their controls, the mean scores of behavior over the 12 weeks were used for analysis. For the infants who died or had to be removed and their controls, the mean scores of behavior prior to death or removal (and an equivalent period of time for controls) were used. Probabilities $<0.05$ were considered statistically significant and probabilities $<0.1$ were considered indicative of statistical trends. All statistical tests were two-tailed.

\section{RESULTS}

A total of 155 episodes of abuse were observed. Four infants suffered severe maternal abuse and either died or had to be permanently removed from their group (three in the 2nd week of life, one in the 5th). Subsequent medical examination or necropsy of the infants did not reveal any clinical condition other than physical trauma. Four infants suffered only mild abuse and survived their first 12 weeks of life without any external intervention. All eight control infants survived without external intervention.

\section{Patterns, Rates, and Contexts of Abuse}

Figure 1 shows the relative proportion of occurrence of different types of abuse. Infant crushing was the most common type of abuse, followed by dragging, stepping on, and throwing. Other types of abuse accounted for less than $5 \%$ of all abuse events. Figure 2 depicts the number of abuse events per hour for the 8 subjects. In most cases, abuse began in the first week and, for the surviving infants, almost disappeared in the third month. In two cases, however, abuse increased in frequency at 5-6 weeks of infant age. Infants suffering rates of abuse of four events per hour or higher died or had to be permanently removed whereas abuse rates lower than four events per hour did not jeopardize infant survival. Severely and mildly abusive mothers did not differ significantly in the relative proportion with which they displayed seven different types of abuse (ANOVA, $F(6,36)=0.27, n s$ ).

To investigate the most common contexts of infant abuse, the social events occurring in the 


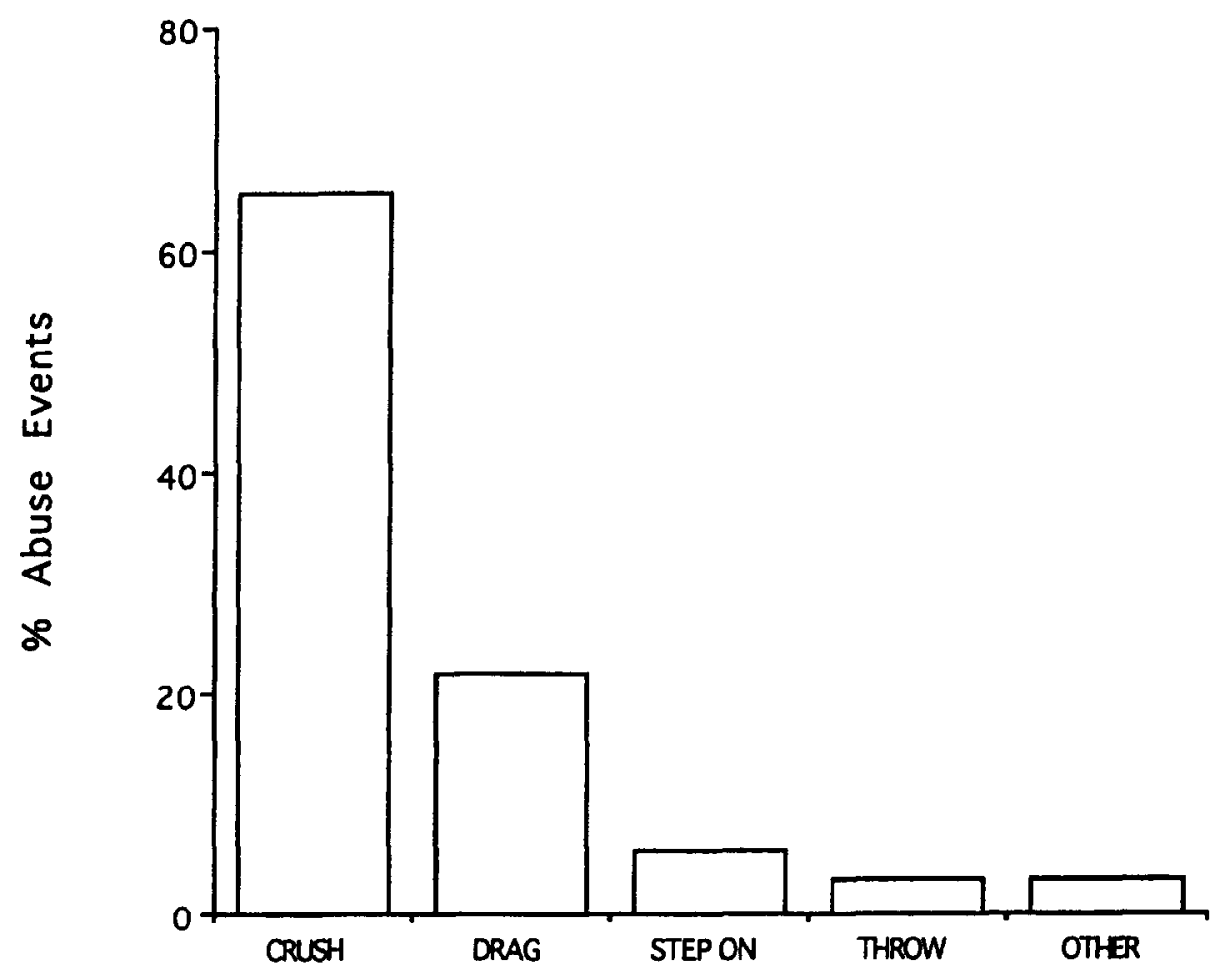

\section{Abuse Patterns}

Ficure 1

Percentage of occurrence of different abuse patterns.

30-s interval prior to the occurrence of abuse were analyzed and classified into one of the following categories: inactivity (no significant events), mother-infant interaction (e.g., change in mother-infant distance, grooming, restraining or rejection), mother-other interaction (e.g., change in mother-other distance, grooming), stress (e.g., aggression received by mother, infant harassment or kidnapping, other conflicts in the group, or extra-group disturbance), and previous infant abuse (i.e., abuse was repeated). For four mothers, the most common context of abuse was stress $(77 \%, 60 \%, 59 \%$, and $31 \%$ of all episodes), for two mothers it was inactivity ( $60 \%$ and $44 \%$ of episodes), and for two mothers it was interaction with others $(80 \%)$ and previous abuse $(48 \%)$, respectively. When data from all abusive mothers were pooled, abuse episodes were distributed by context as follows: $29 \%=$ stress, $26 \%=$ mother-other interaction, $23 \%=$ inactivity, $16 \%=$ previous abuse, and $6 \%=$ mother-infant interaction. Among the most frequent stressful events preceding abuse there were infant kidnapping or harassment, conflicts in the group involving multiple individuals, and extra-group disturbance due to the presence of the veterinarian or the animal caretakers.

\section{Mother-Infant Interactions and Social Behavior}

Normative data on maternal behavior and infant development in pigtail macaques were 


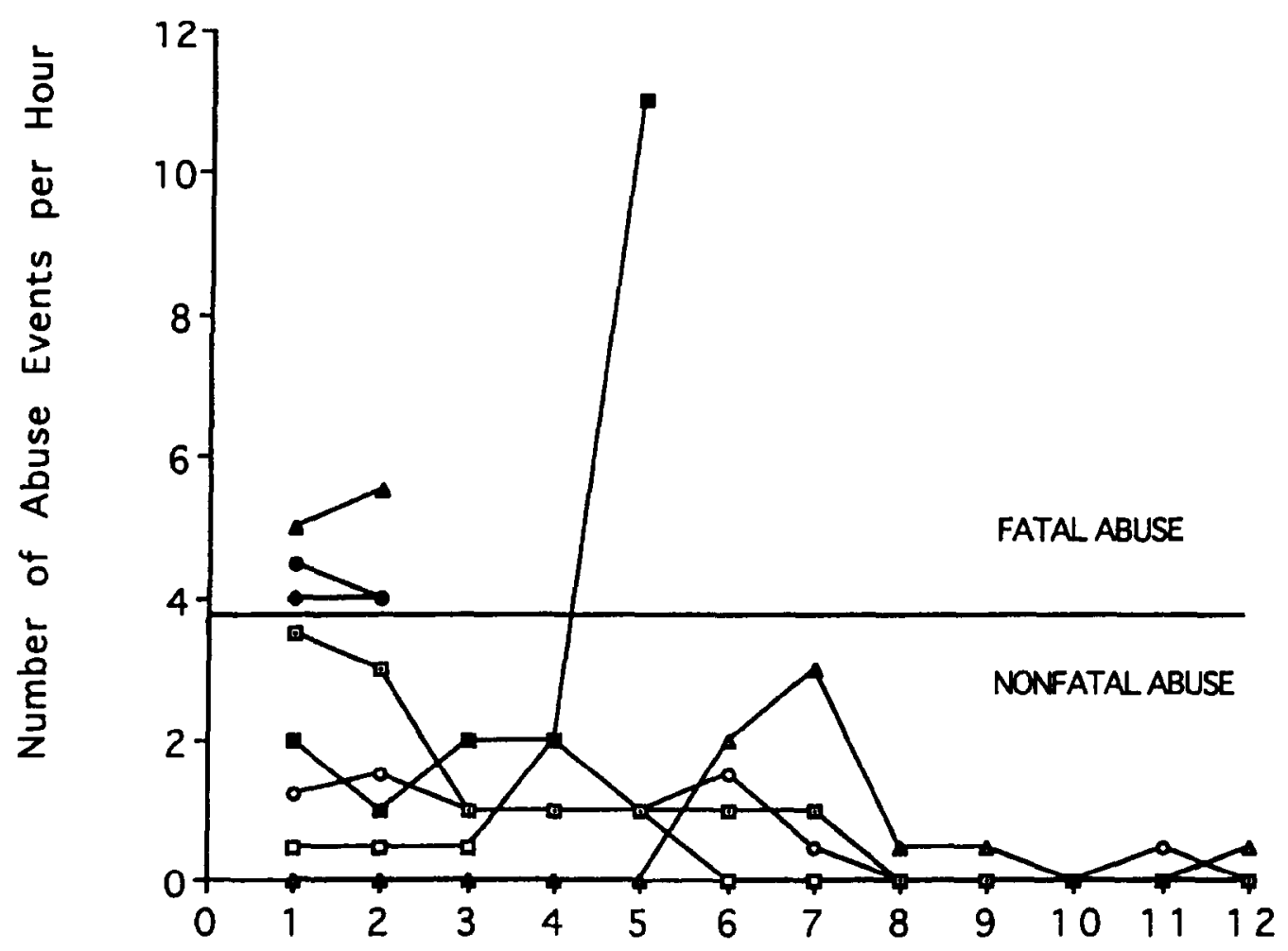

\section{Weeks}

FIGURE 2

Mean number of infant abuse episodes per hour per week for each of the 8 abusive mothers.

presented elsewhere (Maestripieri, 1994b). Abusive mother-infant pairs did not differ significantly from controls in any measure relative to breaking or making contact, approaching or leaving. Similarly, no significant differences were found in the percentage of time spent in mother-infant contact, cradling, or grooming. The abusive mothers whose infants survived tended to score higher than controls in both infant restraining (mean $\pm S E M$, abusive $=17.51 \pm 6.45$, controls $=$ $5.13 \pm 1.35, t(3)=2.53, p=0.08)$ and rejection (abusive $=17.34 \pm 5.64$, controls $=0.72 \pm$ $0.32, t(3)=2.76, p=0.07$ ). Figure 3 shows that most restraining and rejection by mildly abusive mothers were concentrated in the first two months of infant life. When comparisons between subjects and controls were conducted month by month, however, the statistical trends were no longer apparent. Restraining and rejection were too infrequent to be analyzed among mother-infant pairs with fatal abuse.

Abusive mothers did not differ from controls in any social interactions with other group members, including making and receiving contact, grooming, or aggression. Abused infants did not differ from controls in the frequency with which they were handled or harassed by other individuals. In the four mildly abused infants, play with other infants or juveniles made its first appearance at a later age than in controls (mean age in days $\pm S E M$, abused $=46.25 \pm 3.79 ;$ controls $=30.75 \pm$ 

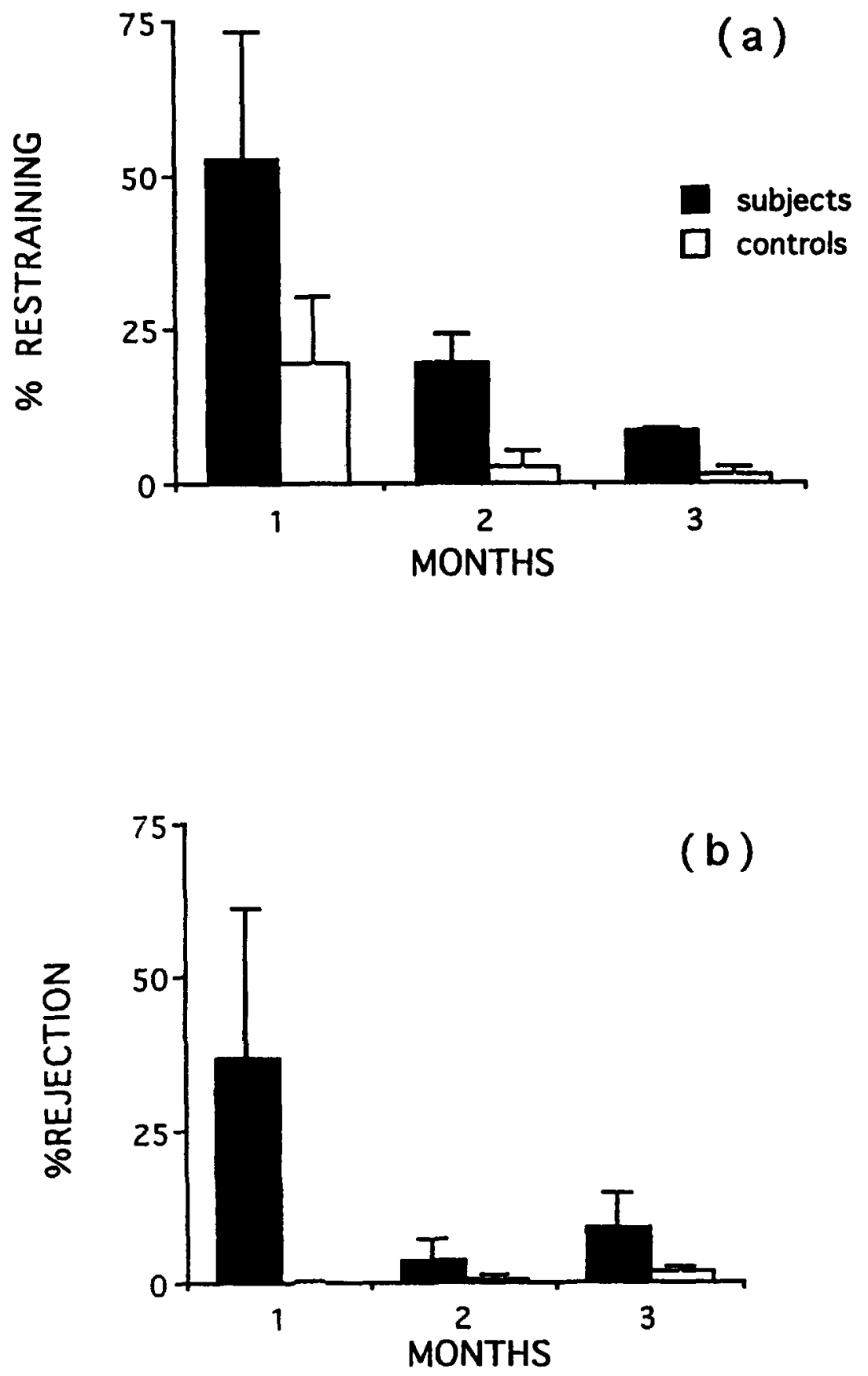

FIGURE 3

(a). Mean ( \pm SEM) percentage of infant attempts to break contact that were prevented by abusive and control mothers (restraining) in the first 3 months of lactation. (b). Mean ( $\pm S E M$ ) percentage of infant attempts to make contact that were prevented by abusive and control mothers (rejection) in the first 3 months of lactation. 
$6.38 ; t(3)=3.95, p=0.03)$ and the total number of play bouts involving abused infants was lower than for controls (abused $=33.25 \pm$ 12.50 ; controls $=46.50 \pm 12.13 ; t(3)=3.02, p$ $=0.05)$. The analysis of play initiation revealed that mildly abused infants initiated play less frequently than controls (abused = $18.25 \pm 7.56$; controls $=25.75 \pm 6.25 ; t(3)=$ $3.38, p=0.04$ ) but play initiated by others did not differ significantly between mildly abused infants and controls (abused $=15.00 \pm 4.95$; controls $=20.75 \pm 6.13 ; t(3)=1.86, n s)$. Play was not observed in the severely abused infants and in their controls because of their young age.

\section{DISCUSSION}

Infant abuse in group-living pigtail macaques appeared as early as the first week of infant life and ceased almost completely by the end of the third month. Three-month-old infants have achieved full locomotor independence from their mothers (Maestripieri, 1996) and therefore are much less vulnerable to abuse. The most common patterns of infant abuse were infant crushing and dragging, followed by stepping on, and throwing. The consequences of abuse ranged from infant distress to serious injury and death. Interestingly, severity of abuse appeared to depend primarily on its frequency of occurrence and not on the physical patterns of abuse. This finding suggests that mild and severe abuse differ from each other quantitatively but not qualitatively.

The most common context of occurrence of infant abuse was stress from the social environment. Stress included infant kidnapping and harassment by other group members, aggression received by the mother, other conflicts in the group, or disturbance outside the group (see also Maestripieri, 1994a). However, since abusive mothers did not receive more aggression than control mothers and their infants were not harassed more than control infants, it seems likely that some abusive mothers are individuals that are particularly vulnerable to stress or that have problems in emotion regulation (Troisi \& D'Amato, 1984). The fact that most abusive mothers consistently abused their successive infants also suggests that environmental contingencies may act in conjunction with individual predispositions in triggering dysfunctional parenting. Findings from other studies suggest that relatedness to other abusive individuals and experience of abuse early in life may be risk factors for abusive parenting as well (Maestripieri \& Carroll, 1998a, b; Maestripieri et al., 1997a, b). In contrast, the fact that abusive mothers alternate abuse with competent maternal behavior suggests that lack of parenting experience does not play an important role in the occurrence of abuse. This is confirmed by the fact that some abusive mothers had successfully reared previous offspring.

Pigtail macaque abusive mothers could not be statistically distinguished from nonabusive mothers in several measures of maternal and social behavior. However, mildly abusive mothers tended to score higher than controls in both infant restraining and rejection. Monkey parenting styles generally vary along the two orthogonal dimensions of Protectiveness and Rejection, and mothers who rank high on both dimensions are classified as Controlling (Fairbanks, 1996). Therefore, the present study lends support to previous findings obtained with rhesus macaques (Macaca mulatta) showing that abusive mothers have highly Controlling parenting styles (Maestripieri, 1998). The association between infant abuse and controlling parenting style suggests that abuse may reflect inappropriate attempts to control the infant's behavior, perhaps in turn linked with maladaptive responsiveness to stress.

The lack of obvious differences in the behavior of abused and control infants suggests that infants do not play an active role in triggering abuse. It cannot be ruled out, however, that abused infants had subtle physical or behavioral handicaps not apparent to human observers, which may have contributed to the 
occurrence of abuse. Mildly abused infants initiated play at a later age, and overall played less frequently, than control infants. Most infant play occurred in the second and in the third month of infant life whereas most abuse was concentrated in the first weeks, thus suggesting that the difference in play may have been a consequence of early abuse. Other studies of infant abuse in individually housed and group-living macaques have shown that abuse increases infant clinging and delays the acquisition of infant independence from their mothers, at least temporarily (Maestripieri, 1998; Ruppenthal et al., 1976). More studies are needed to accurately assess the long-term consequences of early abuse on social and psychological development.

In addition to investigating the developmental consequences of abuse, future studies of this phenomenon in monkeys should also investigate the possible psychological and physiological characteristics that render some mothers vulnerable to displaying abusive parenting. Nonhuman primates offer the opportunity to combine accurate psychological and social investigations with experimental manipulations that would be difficult in humans. Therefore, primate research on infant abuse could make a valuable contribution to the understanding, prevention and treatment of child abuse in humans.

Acknowledgments: This research was supported by grants from the Biomedical Resources Foundation (Houston, TX), the Harry Frank Guggenheim Foundation (New York, NY), and NIH (MH57249) to D.M. and in part by NIH grant RR-00165 to the Yerkes Regional Primate Research Center. The Yerkes Center is fully accredited by the American Association for Accreditation of Laboratory Animal Care.

\section{REFERENCES}

Altmann, J. (1980). Baboon mothers and infants. Cambridge: Harvard University Press.
Fairbanks, L. A. (1996). Individual differences in maternal styles: Causes and consequences for mothers and offspring. In J. S. Rosenblatt, \& C. T. Snowdon (Eds.), Advances in the study of behavior (Vol. 25). New York: Academic Press.

Hinde, R. A., \& Spencer-Booth, Y. (1967). The behaviour of socially living rhesus monkeys in their first two and a half years. Animal Behaviour, 15, 169-196.

Hiraiwa, M. (1981). Maternal and alloparental care in a troop of free-ranging Japanese monkeys. Primates, 22, 309-329.

Maestripieri, D. (1994a). Infant abuse associated with psychosocial stress in a group-living pigtail macaque (Macaca nemestrina) mother. American Journal of Primatology, 32, 41-49.

Maestripieri, D. (1994b). Mother-infant relationships in three species of macaques (Macaca mulatta, $M$. nemestrina, $M$. arctoides). I. Development of the mother-infant relationship in the first three months. Behaviour, 131, 75-96.

Maestripieri, D. (1996). Maternal encouragement of infant locomotion in pigtail macaques (Macaca nemestrina). Animal Behaviour, 51, 603-610.

Maestripieri, D. (1998). Parenting styles of abusive mothers in group-living thesus macaques. Animal Behaviour, 55, 1-11.

Maestripieri, D., \& Carroll, K. A. (1998a). Child abuse and neglect: Usefulness of the animal data. Psychological Bulletin, 123, 211-223.

Maestripieri, D., \& Carroll, K. A. (1998b). Risk factors for infant abuse and neglect in rhesus monkeys. Psychological Science, 9, 143-145.

Maestripieri, D., Wallen, K., \& Carroll, K. A. (1997a). Infant abuse runs in families of groupliving pigtail macaques. Child Abuse \& Neglect, $21,465-471$.

Maestripieri, D., Wallen, K., \& Carroll, K. A. (1997b). Genealogical and demographic influences on infant abuse and neglect in group-living sooty mangabeys (Cencocebus atys). Developmental Psychobiology, 31, 175-180.

Reite, M., \& Caine, N. G. (1983). Child abuse: The nonhuman primate data. New York: Alan Liss.

Ruppenthal, G. C., Arling, G. L., Harlow, H. F., Sackett, G. P., \& Suomi, S. J. (1976). A 10-year perspective of motherless-mother monkey behavior. Journal of Abnormal Psychology, 85, 341-349.

Troisi, A., \& D'Amato, F. R. (1983). Is monkey maternal abuse of offspring aggressive behavior? Aggressive Behavior, 9, 167-173. 
Troisi, A., \& D'Amato, F. R. (1984). Ambivalence in monkey mothering: Infant abuse combined with maternal possessiveness. Journal of Nervous and Mental Disease, 172, 105-108.

Troisi, A., D'Amato, F. R., Fuccillo, R., \& Scucchi, S. (1982). Infant abuse by a wild-born group- living Japanese macaque mother. Journal of Abnormal Psychology, 91, 451-456.

09 June 1997; Revised 28 January 1998 